\title{
Organizational Determinants of Psychosocial Treatment Activity of Providers in VA Mental Health Facilities
}

\author{
Jeffrey A. Alexander ${ }^{1 *}$, Joan R. Bloom ${ }^{2}$, James L. Zazzali ${ }^{2}$ and Kimberly Jinnett ${ }^{3}$ \\ ${ }^{1}$ Department of Health Management and Policy, School of Public Health, The University of Michigan, and Department of Veteran's Affairs, 109 \\ Observatory, Ann Arbor, MI 48103, USA. \\ ${ }^{2}$ Department of Health Policy and Management, University of California, Berkeley, CA, USA \\ ${ }^{3}$ RAND, 1700 Main St, Santa Monica, CA, USA
}

\begin{abstract}
Objective: To identify the determinants of level and intensity of psychosocial treatment activity among staff who deliver services to the severely and mentally ill.

Methods: The study sample consisted of 769 treatment providers working in 77 units in 29 VA mental health facilities. Level of psychosocial care was measured as the number of patient contacts and total hours spent in psychosocial care over a 1 week period. Intensity of psychosocial care was measured as the average time per patient contact. We used hierarchical linear modeling (HLM) to examine the association between level and intensity of care and three categories of determinants-individual provider attributes, work characteristics and treatment setting characteristics.

Results: Providers' occupation is related to both the level and intensity of care. Providers with administrative responsibilities also have fewer patient contacts and lower intensity of such contacts. Providers who perceived their pay and benefits more positively had fewer patient contacts and less intensive patient contacts. Positive relationships with patients and providers were also associated with greater levels and intensity of psychosocial treatment activity among providers. Finally, statistically significant differences in psychosocial treatment activity among units were identified although such differences are not attributable to unit size, patient cohort severity or unit workload.

Conclusions: Level and intensity of psychosocial treatment activity vary systematically by individual attributes of providers, characteristics of the work they perform and attributes of the treatment setting. These factors may provide the basis for designing interventions to modify provider behavior in a manner consistent with emerging financial pressures and treatment modalities for the seriously mentally ill. Copyright (C) 1999 John Wiley \& Sons, Ltd.
\end{abstract}

Received 3 May 1999; accepted 9 November 1999.

\footnotetext{
*Correspondence to: Jeffrey A. Alexander, Department of Health Management and Policy, School of Public Health, The University of Michigan, and Department of Veterans' Affairs, 109 Observatory, Ann Arbor, MI 48103, USA.

Tel.: (734) 936-1194. Fax: (734) 764-4338. Email address: jalexand@umich.edu

Contract grant sponsor: The Seriously Mentally Ill Treatment, Research and Evaluation Center, Ann Arbor, MI

Contract grant sponsor: Center for Mental Health Services Research at the University of California, Berkeley
}

\section{Introduction}

Two fundamental transitions are currently under way in the mental health sector that compel treatment organizations to provide care in different ways and at different levels. First, the psychosocial rehabilitation model of care (as opposed to the medical model) is rapidly gaining prominence as an efficacious method of treating patients and integrating them into the community. ${ }^{1,2}$ This approach to care emphasizes case management, functional skill development and intensive care planning, techniques that require a great deal of individual time and attention from staff who treat patients. To the extent that patients with serious mental illness require more attention to achieve acceptable levels of psychosocial functioning, such efforts are likely to require high levels of psychosocial treatment intensity. At the same time, there has been a movement away from traditional, fee-for-service reimbursement systems toward capitation and other managed care practices..$^{3-6}$ Mental health treatment organizations now operate under strong incentives to keep costs down and productivity high if they are to gain financially. ${ }^{1,3,7,8}$ This has required treatment organizations to attend to issues of efficiency and productivity among treatment staff, particularly in salaried practice settings where practitioner income is not as dependent on output. ${ }^{9}$

Despite these potentially competing pressures to alter the type and level of care provided by mental health treatment staff, there is a general absence of understanding about the 'production process' of mental health treatment programs. ${ }^{10-12}$ The few studies that exist have focused on describing how staff spend their time by documenting work activities of mental health professionals in general, ${ }^{13}$ clinical nurse specialists ${ }^{14}$ and social workers employed in hospital settings. ${ }^{15}$ Although instructive, these studies are largely descriptive and limited to specific types of provider, treatment setting or patient care activity. None, to our knowledge, has addressed psychosocial rehabilitation specifically.

The purpose of this paper is to examine the determinants of level and intensity of psychosocial treatment activity among staff responsible for delivering mental health services 
to the chronically mentally ill. Results of the study are intended to identify those individual, work and treatment setting attributes that are most salient in determining psychosocial treatment activity and thus, those that represent potential leverage points for changing such behavior.

\section{Conceptual Framework}

Our focus in this paper is on psychosocial rehabilitation and the treatment activities associated with that mode of care. Psychosocial rehabilitation has its roots in multiple disciplines and professional domains, including psychiatry, psychology, nursing, social work and occupational rehabilitation therapy, among others. ${ }^{16}$ The goal of psychosocial rehabilitation is not only to treat the symptoms of serious mental illness (SMI), but also other, non-clinical consequences of SMI. Psychosocial rehabilitation focuses on how disabilities and disadvantages affect the functioning of the individual. Those who practice psychosocial rehabilitation assume that decreases in symptoms do not necessarily lead to increases in functioning (and vice versa). Furthermore, the transition from institutionalization to community-based care and living requires individuals with SMI to develop skills different from those emphasized in institutional settings or prevented from developing because of their illness. ${ }^{17-19}$

Psychosocial treatment activities usually involve the patient directly, as in therapy sessions, but may also use more indirect methods such as case management and care planning. In particular, we consider scheduled treatment activities provided for a patient, as planned and recorded by the multi-disciplinary treatment team. Specifically, these activities may fall under several categories, including direct patient care (e.g., psychotherapeutic interventions, psychosocial skill building and development, behavioral interventions and biological/psychological interventions such as relaxation and movement therapy); diagnosis, evaluation and treatment planning; brokerage, linkage and advocacy; discharge planning and placement; liaison with family and community and leisure activities. Because they are not considered psychosocial rehabilitation, we exclude from psychosocial treatment care related to activities of daily living (e.g. bathing, dressing, feeding), diagnosing and treating physical conditions, administration of medication, spontaneous interventions that may occur throughout the day and non-treatment activity.

Psychosocial treatment activity may be influenced by a number of factors, ranging through the context in which care is provided, characteristics of the work itself or personal attributes of the staff member. Rather than focusing on one type of determinant (e.g. financial incentives) we propose a multidimensional model that assumes that potential determinants of treatment activity are complex and emanate from multiple sources. Further, treatment activity itself can be conceptualized and measured in different ways. We focus in this paper on two dimensions of psychosocial treatment activity: (i) level of activity (productivity) and (ii) intensity of activity. Level of activity is defined as the amount of psychosocial care a staff member provides over a defined period of time. Intensity of psychosocial treatment activity refers to the amount of time a staff member spends in each patient treatment contact. These dimensions may be negatively correlated in that intensity of treatment activity (spending more time with each patient) will often preclude high levels of psychosocial treatment activity (seeing more patients). Each of our three categories of treatment activity predictors is discussed below in terms of its potential relationship to both the level and intensity of psychosocial treatment activity.

\section{Individual Provider Attributes}

Individual treatment providers bring to their work a variety of experiences, roles and professional orientations that may influence their psychosocial treatment activity independent of the work conditions or the treatment setting in which work is performed. For example, recent evidence suggests that nursing personnel with different training, demographic attributes and education react very differently to working conditions and that these conditions will in turn affect their intention to quit and actual quitting behavior. ${ }^{20}$ By extension we assert that treatment personnel may be predisposed to provide higher or lower levels of psychosocial treatment, or more or less intensive psychosocial treatment as a function of their training (occupation), what roles they are assigned in the treatment setting and the amount of experience they possess in the organization. For example, because of the emphasis on developing social and functional skills (as opposed to psychotherapy), a psychiatrist might be expected to spend less time with any one patient (less intensive treatment) under the psychosocial rehabilitation model, but registered nurses may spend more time with individual patients as they help them to develop functional skills. Similarly, a staff member whose role includes administrative duties might spend less time and have fewer contacts with patients than one whose role is defined as $100 \%$ patient care. Finally, more experienced treatment staff may have developed more efficient routines over time to allow them to spend more time caring for patients (as opposed to other duties). We anticipate that individual attributes of providers will affect level and intensity of psychosocial treatment activity independently of work conditions or contextual features of the treatment setting.

\section{Work Characteristics}

There is considerable evidence in the economic, industrial psychology and industrial engineering literatures that conditions under which work is performed will affect how work is performed. There is, for example, strong evidence to suggest that reward systems based on piece work, task specialization and close supervisory control relate to increased employee productivity. ${ }^{21}$ However, because mental health delivery is both multidisciplinary and technologically less precise than manufacturing, findings from other types of organization may not apply. We adopt the perspective of examining dimensions of an individual's work conditions 
(e.g. workload, hazards, pay and rewards) that are specific to mental health delivery and their relationship to psychosocial treatment activity. We assume that such conditions are experienced differently by individuals rather than representing universal effects, and that some characteristics of work will be more important than others in terms of their salience on level and intensity of psychosocial treatment activity. Six dimensions of work are considered: (i) the degree to which a treatment staff member has positive professional relationships with patients, (ii) the degree to which a treatment staff member has positive working relationships with co-workers, (iii) appropriateness/fairness of job related pay and rewards, (iv) level of job-related physical hazards, (v) how well roles and expectations for the treatment staff member's job are defined and (vi) the degree to which workload precludes effective delivery of psychosocial treatment. In general, we anticipate that positive relationships with patients and co-workers will result in greater patient contact, as will perceptions of fairness of pay and rewards and the clarity of treatment staff's roles and expectations. Conversely, the level of job-related physical hazards and a heavy workload will be inversely related to level and intensity of psychosocial care contact.

\section{Treatment Setting Characteristics}

Beyond the effects of individual attributes and work characteristics on psychosocial treatment activity, we hypothesize that the context in which treatment work is performed will independently influence such activity. A provider's immediate work environment structures the rate, processes and content of interactions with other providers and with patients, collectively referred to as the demands of care. ${ }^{22}$ Context, in other words, regulates what individual providers do in the course of their day to day activity. The study examines three potentially important contextual attributes of the treatment setting - unit size, functional status of patient cohort and unit workload. For example, treating groups of poorly functioning patients may lower levels but raise intensity of psychosocial treatment activity. Such patients require more individualized attention, which may preclude providers from seeing a greater number of patients.

\section{Methods}

\section{Study Setting}

This analysis was conducted as part of a larger study, whose purpose was to evaluate the US Department of Veterans' Affairs (VA) Long-Term Mental Health Enhancement Program (LTMHEP). The goal of the LTMHEP was to improve the quality of psychosocial and medical care in the VA's 29 long-term neuropsychiatric hospitals. These hospitals are distributed across the United States and their primary mission is to provide long-term psychiatric care to veterans with mental illness. These facilities are similar in size, location (all located in rural or suburban areas) and services (both inpatient and outpatient); none are part of academic medical centers. As part of the evaluation of the LTMHEP, we conducted an assessment of both patients and staff in large number of units in the 29 VA hospitals. Both inpatient and outpatient units were eligible for inclusion in the study group if they met the following conditions: (i) $50 \%$ or more of the patients treated by the unit were diagnosed with a psychosis (e.g. schizophrenia, major mood disorder or dementia) and (ii) there was documented evidence of those patients suffering from psychosis having a cumulative length of stay in a VA psychiatric unit of at least 150 days in the past year, or five or more admissions to VA psychiatric units. These criteria were applied to identify those units whose patients were predominantly the seriously mentally ill (SMI). Both inpatient and outpatient units were considered under these criteria without regard to size of unit.

\section{Sample}

The primary sample for this investigation was 769 treatment staff responsible for delivering care to patients with serious mental illness (SMI) in 29 VA mental health facilities. From the 29 facilities, 77 inpatient and outpatient units delivering such care were selected for inclusion in the study based on the following criteria: (i) patient mix, (ii) funding under a special VA long-term mental health enhancement program and (iii) achieving a balance of treatment staff representation between inpatient and outpatient units. Within the 77 units chosen for study, all treatment staff were sampled. Treatment staff included those individuals who provided some form of direct patient care to patients in those units. These staff included psychiatrists, psychologists, social workers, recreational therapists, occupational therapists, nurses, nurses' aides and nursing assistants.

\section{Data Sources}

Data employed in this study were obtained from multiple sources and collected under the auspices of the VA's Serious Mental Illness Treatment Research and Evaluation Center (SMITREC). The primary instrument for collecting psychosocial treatment activity data was the patient contact survey. 944 out of the 1117 treatment staff assigned to the 77 sample patient care units completed this encounter-based instrument. The survey was completed in sample units over a 1 week period in April 1994. This period was assumed to be long enough to capture variation in the work patterns of treatment staff, yet short enough to ensure accurate recording and a high response rate. Treatment staff were asked to record all patient contacts that conformed to our definition of psychosocial treatment activity during that period including the length, type, location and content of the contact. SMITREC staff performed data entry and measurement development using contact information. Surveys were considered usable if the respondent reported at least one patient contact during the reporting period and confined patient contacts to one patient care unit. This resulted in a final usable sample from the patient contact survey of 916 providers. 
The second source of data for the study was the staff Job Satisfaction Survey (JSS). This survey was administered to all treatment staff in 129 treatment units (including the sample units) in 1994 and 1995. The JSS survey administration was lagged by 4 months to the patient contact survey in order to strengthen assumptions of causality between independent and dependent variables. The survey contained items related to perceptions of working conditions, treatment team relations, job satisfaction and respondent demographics. This survey received a response rate of $97 \%$.

Administrative databases were used to capture information on type of unit, functional status of patients in a unit and FTE assignment of staff members to a unit. Data from all sources were merged on a special provider code. The final, usable sample was 769 treatment staff (an overall patient contact survey response rate of $69 \%$ ).

\section{Measures}

Psychosocial treatment activity was captured by three measures. The first, number of patient contacts, was a simple count of all psychosocial treatment encounters by a staff member over the one-week recording period, regardless of the type, location or nature of the contact. Multiple contacts with the same patients were recorded as separate contacts for purposes of constructing this measure. The second measure, number of psychosocial care hours, was computed as the total time consumed by all psychosocial care contacts during the reporting period. These two measures assessed level of psychosocial treatment activity. The third measure captured intensity of psychosocial care contacts and was computed as the average length of all contacts over the reporting period. Because the empirical distributions of these measures were not normal, a natural log transformation was taken. Visual inspections of the distributions of the transformed variables suggest that all three more closely approximate normal distributions. Our measures of psychosocial treatment activity conform to the standard of service output used by other investigators ${ }^{9,23}$ - the amount of direct services provided, expressed either in hours or number of patients served.

(i) Individual Provider Characteristics. Five individuallevel variables were included in the model as predictors of level of psychosocial treatment activity: full time equivalent assignment to unit, administrative responsibility, tenure in the VA facility, occupation and gender. FTE assignment was measured as the proportion of time the treatment staff member was assigned to the focal unit. Administrative responsibility was a categorical variable (0/1) that identified whether or not a treatment staff member had administrative responsibilities in the focal unit. VA tenure was measured as the number of years and fraction thereof that the staff member had been working at his/her current VA facility. Gender was coded as $1=$ male, $0=$ female. Finally, occupation was coded as a series of dummy variables representing the following occupations: psychiatrist, psychologist, social worker, registered nurse, occupational and recreational therapists, LPNs/nurses' aides and other. Because LPN/nurses' aides represented the largest occupational group, they were used as the reference category in the regression models.

(ii) Work Characteristics were measured by six, multiitem scales that captured different dimensions of treatment staff working conditions: relationships with patients, relationships with co-workers, workload, pay and rewards, physical hazards and role clarity. These scales were derived from multiple items in the JSS survey, using principal components factor analysis and reliability assessments of the resulting factors. All scales achieved a Cronbachs' reliability coefficient of .70 or higher. Scales were computed as the average score across all items in the scale. Items were coded on a 1-7 agree-disagree continuum. Some items were reverse coded so that high scale scores reflect more positive perceptions of a particular work characteristic. Scale items are presented in the Appendix.

(iii) Treatment Setting Characteristics. Three unit level characteristics were measured: unit workload, unit size and unit case-mix severity. Unit size was the number of treatment staff assigned to the unit at the time of the JSS was administered. Because our sample contained relatively few part-time staff, we did not weight them differently from full time staff in calculating unit size.

Unit case-mix severity was based on functional ability of the patient cohort in each unit. Functional ability represents the average level of psychological, social and occupational functioning of patients treated in the unit. It was measured as the weighted mean of the proportion of patients on the unit who were in each of five categories of the Global Assessment of Functioning Scale (GAF). Higher scores indicate less severe impairment of the patient cohort. ${ }^{24}$ Although some have expressed concerns regarding the reliability of the GAF for evaluating individual patients, ${ }^{25}$ our use of the scale as the basis for characterizing a group of patients may reduce sensitivity to any unreliable assessments.

Unit workload was based on the average daily census (for inpatient units) or the number of outpatient visits (for outpatient programs) during a randomly selected week in April 1994. To standardize the measure for both inpatient and outpatient units, we created separate $Z$-scores for the two types of unit. Thus the workload measure represents a unit's deviation from the mean workload for all units of similar type.

Descriptive statistics and separate correlation matrixes for the measures of individual-level characteristics and unitlevel characteristics are presented in Table $\mathbf{1}$. 


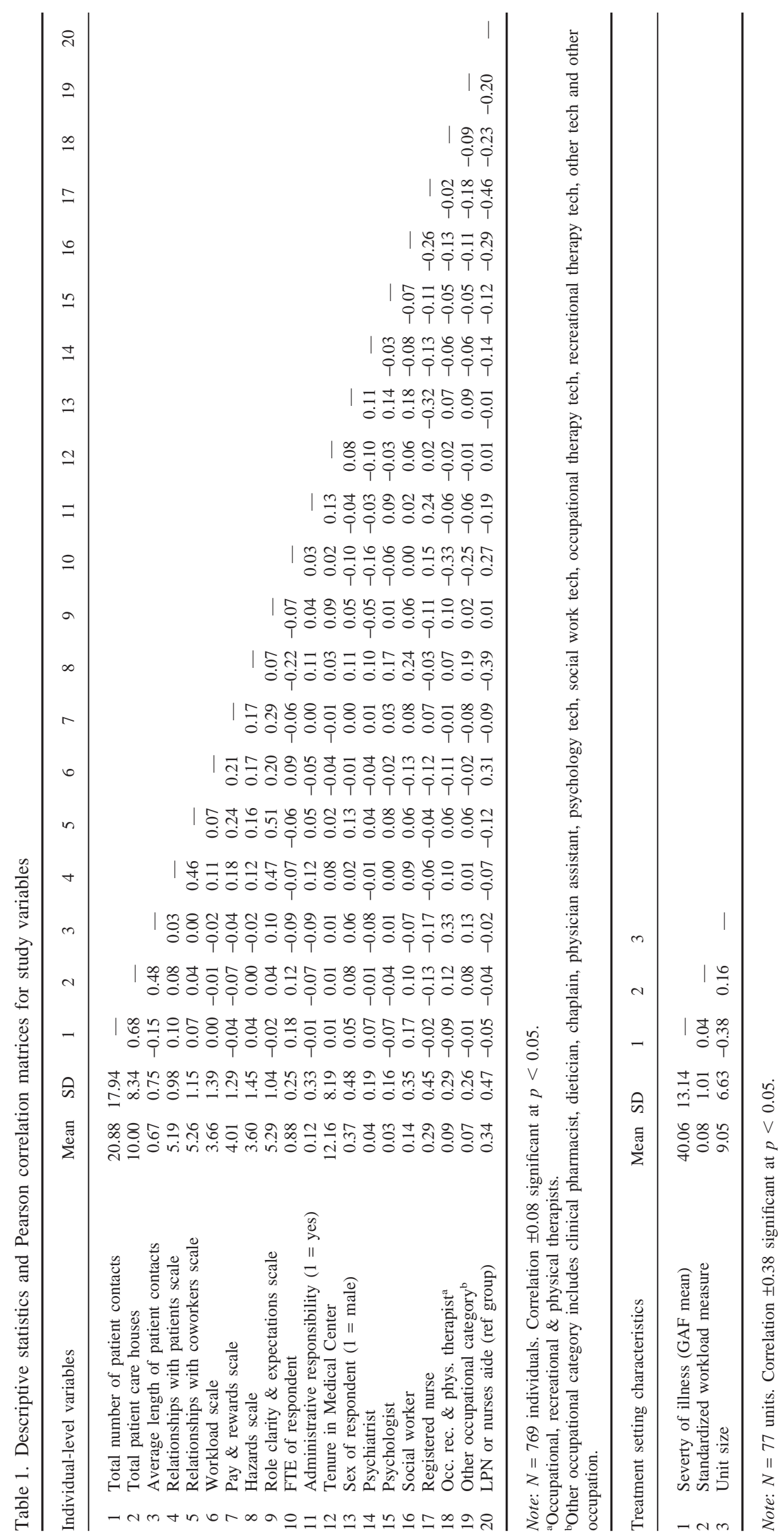




\section{Analyses}

Our conceptual model explains provider level of activity using predictors at two different levels of analysisindividual staff member and treatment setting or program. These attributes are structured hierarchically because individual treatment staff work within units or programs, and unit or program characteristics were assumed to affect treatment staff of the unit similarly. Conventional analytic approaches such as assigning the same unit value to all treatment staff in that unit are inappropriate because they do not account for the lack of independence among observations in a given unit. $^{26,27}$ This situation requires a multivariate analytic technique capable of accounting for the multilevel structure of the data in determining regression coefficients and standard errors.

We used hierarchical linear modeling (HLM) to examine the effects of unit-level and provider-level predictors on level of psychosocial treatment activity. This procedure adjusts for varying unit-level characteristics by appropriately separating out within-unit variance from between-unit variance. ${ }^{28,29}$ Specifically, the first stage of the analysis estimates the within-unit parameters. These parameters can be random (allowed to vary randomly across units) or fixed (set as controls with no parameter variance across units). At the second stage, only between-unit variation in the random parameters is analyzed. The final stage involves examining the explanatory power of between-unit variables on the within-unit random parameters (intercepts and slopes) produced as part of the first stage analysis.

\section{Results}

Descriptive analysis indicates considerable variation in psychosocial treatment activity among our sample of providers. They averaged 21 patient contacts over the reporting period $(\mathrm{SD}=18)$. The minimum number of contact was 1 and the maximum was 113. Total psychosocial treatment hours displayed similar variation. Sample members provided an average of 10 hours of psychosocial treatment (SD = 8.3 ) with a minimum of 0.25 and a maximum of 60 hours. Finally, average length of treatment was 40 minutes $(\mathrm{SD}=$ 0.75 ) with a minimum of 12 minutes and a maximum of 7.5 hours.

The intraclass correlation indicates that a significant proportion of the variance in psychosocial treatment activity occurs across units. This finding holds for all three dependent variables. The percent between unit variance was $20.3 \%$ for total patient care hours, $28.4 \%$ for average length of patient contacts and $21.3 \%$ for total number of patient contacts. These results support the use of HLM in order to adequately control for the effects of membership in a unit or program on the dependent variable, and to assess the effects of unitlevel variables on level of psychosocial treatment activity. Although results of the first stage analysis revealed significant slope variance for several of the independent variables, the associated fixed effects of these variables were not significant when the slopes were allowed to vary randomly.* We elected therefore to pursue random intercept, fixed slope multilevel models.

Results of the final multilevel model using fixed slopes and random intercepts are presented in Table 2. Findings for each dependent variable are discussed separately.

\section{Number of Patient Contacts}

Number of patient contacts reflects the frequency of psychosocial treatment interactions with patients on a unit or program by a staff member. None of the unit level predictors was significantly related to the dependent variable. Five individual provider attributes emerged as significant predictors of number of patient contacts. As expected, the higher the provider's FTE assignment to the unit, the greater the number of patient contacts. In other words, more time spent on the unit by the provider translates into more contacts with patients. Results also indicated a negative and significant coefficient for administrative responsibility. Those providers who hold administrative positions in their unit or program engage in fewer patient contacts than their colleagues who do not hold such positions. Several differences in number of patient contacts were found by occupational category. Relative to LPNs/nurses aides, psychiatrists, social workers and registered nurses had significantly more patient contacts during the observation period, controlling for all other individual and unit level variables. No statistically significant association between organizational tenure or gender and number of contacts was observed. The model explained $12 \%$ of the variance in the dependent variable.

Three of the six measures of work characteristics were significantly associated with number of patient contacts. As expected, the more positive the provider's relationship with co-workers and the more positive his/her relationship with patients, the greater the number of patient contacts. Contrary to expectations, however, greater pay and rewards was significantly and negatively related to number of patient contacts, indicating that providers who perceived themselves to be well paid and appropriately recognized and rewarded for their work engaged in fewer patient contacts than their colleagues who felt less positively about pay and rewards. Role clarity, physical hazards and workload were not significantly associated with number of patient contacts.

Because our measure of patient contacts includes multiple contacts with the same patient, a case could be made that this inflates the level of activity of a provider and may represent a qualitatively different type of activity than a measure based on unique patient contacts. To explore this possibility we ran the same model using the lognormal transformation of number of unique contacts as the dependent

\footnotetext{
*The amount of total time that psychiatrists and registered nurses spend with clients varied significantly across units, as did the average length of time per contact for therapists and other occupations. However, the main effects of these occupational groups became non-significant when these occupational group slopes were allowed to vary randomly across groups. This pattern of findings suggests the need for further exploration of occupational group slope variance by units.
} 
Table 2. Final hierarchical linear model: the effects of unit and individual-level variables on level and intensity of psychosocial treatment

\begin{tabular}{|c|c|c|c|c|c|c|}
\hline \multirow[t]{2}{*}{ Predictor variable } & \multicolumn{2}{|c|}{$\begin{array}{l}\text { Natural log of number of } \\
\text { patient contacts }\end{array}$} & \multicolumn{2}{|c|}{$\begin{array}{l}\text { Natural log of patient care } \\
\text { hours }\end{array}$} & \multicolumn{2}{|c|}{$\begin{array}{l}\text { Natural log of average } \\
\text { length of patient contacts }\end{array}$} \\
\hline & Effect & $S E$ & Effect & $S E$ & Effect & $S E$ \\
\hline Overall intercept & $2.398 * *$ & 0.279 & $1.626 * *$ & 0.266 & $-0.688 * *$ & 0.193 \\
\hline \multicolumn{7}{|l|}{ Treatment setting characteristics } \\
\hline Severity of illness (GAF mean) & 0.006 & 0.005 & 0.010 & 0.005 & 0.005 & 0.004 \\
\hline Standardized workload measure & 0.051 & 0.064 & 0.100 & 0.061 & 0.071 & 0.044 \\
\hline Unit size & 0.003 & 0.010 & -0.005 & 0.010 & -0.011 & 0.007 \\
\hline \multicolumn{7}{|l|}{ Work characteristics } \\
\hline Relationships with patients scale & $0.093^{*}$ & 0.041 & 0.040 & 0.041 & -0.042 & 0.025 \\
\hline Relationships with co-workers scale & $0.069 *$ & 0.036 & 0.041 & 0.037 & $-0.051 *$ & 0.022 \\
\hline Workload scale & 0.024 & 0.029 & 0.007 & 0.029 & -0.023 & 0.018 \\
\hline Pay \& rewards scale & $-0.061 *$ & 0.029 & $-0.069 *$ & 0.029 & -0.003 & 0.018 \\
\hline Hazards scale & 0.004 & 0.031 & -0.006 & 0.031 & -0.004 & 0.019 \\
\hline Role clarity \& expectations scale & -0.073 & 0.041 & -0.021 & 0.041 & $0.069 * *$ & 0.025 \\
\hline \multicolumn{7}{|l|}{ Individual attributes } \\
\hline FTE of respondent & $1.154 * *$ & 0.171 & $1.298 * *$ & 0.174 & $0.258 * *$ & 0.106 \\
\hline Administrative responsibility $(1=$ yes $)$ & $-0.250 *$ & 0.107 & $-0.386 * *$ & 0.109 & $-0.141^{*}$ & 0.066 \\
\hline Tenure in VA medical center (years) & 0.004 & 0.004 & 0.004 & 0.004 & 0.002 & 0.003 \\
\hline Sex of respondent $(1=$ male $)$ & 0.018 & 0.072 & 0.036 & 0.073 & -0.001 & 0.045 \\
\hline Psychiatrist & $0.705^{* *}$ & 0.193 & $0.379^{*}$ & 0.196 & $-0.403 * *$ & 0.119 \\
\hline Psychologist & 0.205 & 0.232 & 0.420 & 0.236 & 0.234 & 0.143 \\
\hline Social worker & $0.668 * *$ & 0.148 & $0.610 * *$ & 0.151 & -0.109 & 0.092 \\
\hline Registered nurse & $0.198 *$ & 0.091 & 0.054 & 0.092 & $-0.197 * *$ & 0.056 \\
\hline Occ. rec. \& phys. therapist & 0.249 & 0.146 & $0.697 * *$ & 0.149 & $0.568 * *$ & 0.090 \\
\hline Other occupation & 0.154 & 0.163 & 0.255 & 0.165 & 0.089 & 0.101 \\
\hline \multicolumn{7}{|l|}{ Model summary } \\
\hline$\%$ of variance explained by final model & 12.000 & & 19.000 & & 24.000 & \\
\hline
\end{tabular}

Note: LPNs and nurses' aides constitute the occupation reference group.

${ }^{*} p<0.05$. $* * p<0.01$.

variable. Results (available from authors) were identical to the original model except that administrative responsibility and registered nurses were no longer significant.

\section{Total Psychosocial Care Hours}

Examination of total number of psychosocial care hours allows for the possibility that, although patient contact interactions may be relatively low, time spent in psychosocial care overall may be high. Findings from the HLM model predicting number of psychosocial care hours generally paralleled those of the number of patient contacts model, particularly for the effects of the individual provider attributes and unit level variables. Specifically, FTE assignment was positively related, and administrative responsibility negatively related to number of psychosocial care hours. Psychiatrists, occupational and recreational and physical therapists and social workers, relative to LPNs/aides, provide more psychosocial care hours. Registered nurses did not differ significantly from LPNs/aides in the number of psychosocial care hours provided. Overall, the model accounted for $19 \%$ of the explained variance in treatment hours.

As in the previous model, the unit-level predictors displayed no association with number of psychosocial care hours provided when all other individual provider and work characteristics were controlled.

Only one of the six measures of work characteristics was significantly related to number of psychosocial care hours provided. As in the first model, pay and rewards was negatively and significantly associated with the dependent variable. Thus, positive pay and rewards experienced by treatment staff members are associated with a lower number of psychosocial care hours than their colleagues who experience less satisfactory pay and rewards.

\section{Average Length of Contact}

Average length of contact is a measure of intensity or depth of patient contact interactions. The longer the contact, the more intense the provider's interaction with the patient. Findings from this model again indicate no effects of the three unit level variables on the dependent variable. FTE assignment has a positive and significant effect on average length of contact and administrative role has a negative effect on this dependent variable. Psychiatrists and registered nurses (relative to LPNs/aides) have shorter average contacts with patients. Occupational and recreational therapists, 
by contrast, exhibit longer average patient contacts than LPNs/aides. No differences in average length of patient contact were found between LPNs/aides, on the one hand, and psychologists and social workers, on the other.

Two work characteristics displayed a statistically significant relationship with the dependent variable. In contrast to model 1, which predicted number of patient contacts, relationship with co-workers displayed a negative and significant association with average length of patient contacts. That is, the more positive relationships with coworkers the shorter the average length of contact. Role clarity was positively and significantly related to average length of patient contacts. In other words, staff who experience high levels of role clarity and expectations in their jobs tend to engage in more intensive psychosocial care contacts than those whose work roles are less well defined. The model accounted for $24 \%$ of the variance in average length of contact.

\section{Discussion}

Five findings from this study are of particular relevance. First, occupation of the provider is consistently related to both the level and intensity of care provided. Different types of provider engage in different levels of care, even controlling for work characteristics, treatment setting characteristics and other individual attributes. Second, the assignment of administrative responsibilities to treatment staff, over and above their patient care duties is associated with fewer patient contacts and lower intensity of such contacts. Third, treatment staff who view their pay and benefits more negatively tend to have more frequent patient contacts and to spend more time in psychosocial care. Fourth, demands of patient care work (e.g. work hazards and workload) exercise less influence on level and intensity of psychosocial care than factors that define expectations of providers jobs and the relationships between providers and other staff and patients. Fifth, significant differences in level and intensity of psychosocial treatment activity exist among treatment settings, although such differences are not well explained by case mix, workload or unit size.

Occupation was found to be a significant predictor of all three measures of level and intensity of psychosocial care. This pattern of findings suggests that different roles operate in the delivery of care under the psychosocial rehabilitation model.$^{30}$ Each discipline contributes to psychosocial care in different ways and, therefore, should be subject to different standards of productivity. In the context of increasing pressures imposed by managed care and the psychosocial rehabilitation model, the question may not be whether different members of the treatment staff provide different levels and intensity of care to their patients but the extent to which variation in these psychosocial treatment behaviors exist within a particular occupational category (e.g. RNs, social workers). Indeed, the significant slope variance we found reveals that several occupational groups (psychiatrists, RNs, therapists and other occupational groups) engage in different levels and intensity of psychosocial treatment across units. In other words, these occupational groups provide higher levels of psychosocial treatment activity on some units than they do on others. Understanding why this is the case warrants further examination. The finding that psychiatrists, occupational and recreational and physical therapists and social workers spend more hours providing psychosocial care compared to nurses and vocational nurses appears, at first, to be counter-intuitive. However, our definition of psychosocial rehabilitation does not include purely custodial activities, which may account for these differences. However, to the extent that activities of daily living consist of skills development, they are classified under case management and are considered an element of psychosocial rehabilitation.

A key finding of this study is that work and job conditions make a difference for both level and intensity of psychosocial care, even controlling for all other treatment setting and individual attributes. Regardless of whether the provider is a psychiatrist, social worker, registered nurse or nursing assistant, the conditions under which psychosocial care work is performed affect level and intensity of care independently. Somewhat surprisingly, the characteristics associated with the 'demands' of work apparently exercise little impact on the level and intensity of care. For example, neither perceptions of workload nor physical hazards encountered on the job influence these two dependent variables. Similarly, objective measures of workload and severity of illness among the patient cohorts in a treatment setting did not influence level or intensity of care provided. These findings are important insofar as they suggest that treatment staff have the ability to work around or through such externally imposed demands on their time. More important are factors that define role expectations and the relationships between staff and patients on the one hand or staff and co-workers on the other. These relationship factors tend to speak to the importance of the coordination of care required under the psychosocial rehabilitation model and the importance of intrinsic satisfaction in the work setting as a factor explaining level and intensity of care.

The inverse relationship between providers' positive perceptions of pay and benefits and level of patient care activity was unexpected and runs counter to recent research that suggests that more satisfied employees are more productive. ${ }^{31}$ Two explanations for this counter-intuitive finding are offered. First, providers who are dissatisfied with their pay and benefits may work more in the hope that increasing their productivity will be recognized and rewarded. Alternatively, productive providers who feel they are not adequately rewarded may see themselves as relatively deprived. That is, providers who spend long hours in providing care to their patients may feel that the rewards they are receiving are inadequate relative to their efforts, especially when compared to their colleagues. The converse is also possible: staff spending more time in supervision and administrative activities have greater pay and rewards and, by virtue of their positions, reduced time for direct contact with patients. To explore this line of argument we reran the models to include all possible interaction terms 
between pay and rewards, and our six occupational categories. Significant interaction effects would suggest that particular occupations are more or less sensitive to pay and rewards as a motivator for level of activity. Results of these analyses (available from the authors) produced only one significant finding among the 18 interaction terms examined. The interaction of social workers with pay and rewards was positively related to average length of patient contacts.

Although the treatment setting variables in our model (e.g. unit size, unit workload) were not statistically significant predictors of level or intensity of psychosocial care, HLM results did indicate significant differences in all three dependent variables across units. This means that there are unmeasured attributes specific to the treatment settings that affect the level and intensity of psychosocial treatment activity provided by treatment staff in those units. Future research should examine characteristics such as unit culture, treatment philosophy, leadership or interorganizational dependence as factors that might influence the level and intensity of psychosocial treatment provided by individual providers. ${ }^{32-34}$

The association among our three measures of level and intensity of care also has implications for how work is organized in treatment settings in order to maximize productivity and intensity of care under the psychosocial rehabilitation model. As expected, the association between number of patient contacts and total psychosocial care hours is strongly and positively correlated at $r=0.68$. Somewhat unexpectedly, however, number of patient contacts and average length of contacts are only modestly associated ( $r$ $=-0.15)$. This suggests that there is not a tradeoff between intensity of care and level of care provided in long term treatment settings. In fact, both number of patient contacts and intensity of contacts appear to be driven by the total amount of time providers spend in psychosocial care. Treatment organizations need to address issues of reducing non-treatment responsibilities on treatment staff to allow them to maximize the time they have available to engage in care. This interpretation is further supported by our results that administrative responsibilities of treatment staff result in reduced levels of patient contact and lower intensity of care. Strategies to effect this change might include centralizing administrative functions within the treatment setting so that administration becomes a full time activity. Under this scenario, administrators would be freed of potentially conflicting care demands, just as caregivers would be freed of conflicting administrative responsibilities. Finally, improving coordination among providers within and across treatment units would increase treatment staff access to the resources, personnel and facilities necessary to effectively apply the psychosocial rehabilitation model. Level and intensity of care apparently are not mutually inconsistent ends. Concerns about standards of psychosocial treatment being reduced due to pressures to increase productivity (or level of care) may be exaggerated. To the extent that work conditions can be improved and providers enabled to spend more of their time in psychosocial treatment, both goals might be realized.
A potential limitation of our study relates to our inability to assess patient severity differences by individual provider. To the extent that the severity of patients treated by different providers within a unit varies, we may not have appropriately adjusted for patient severity in our assessment of psychosocial treatment activity. Several factors, however, militate against this possibility. First all units in the sample were selected on the basis of their case mix - each unit treats patients suffering predominantly from SMI, thus providing a relatively homogeneous patient population. Second, psychosocial treatment on the sample units is provided via a multidisciplinary treatment team. This team based approach to care, by definition, is designed to allow specialists in different facets of care to coordinate and plan treatment for the entire group of patients for which it is responsible. Each team member specialist provides services to all patients requiring those services, not a select cohort. While it is clearly the case that treatment intensity will vary by the type of service provided, we have controlled for occupation to account for this.

A second limitation of the study is that our measures focus on level and intensity of activity and do not consider the quality of psychosocial treatment encounters between treatment staff and patients. This question of quality of care undoubtedly will surface in policy discussions related to cost savings and staff productivity. In order to address the quality issue, future research should consider using methods such as audio or videotapes of sample provider-patient interactions to supplement the methods used in the current study.

To what extent can these findings be generalized internationally? In most countries outside of the USA, mental health services are financed or delivered (or both) in the public sector. Canada and the United Kingdom are two examples of publicly financed and delivered health systems in the West. ${ }^{35}$ Countries in Asia (e.g. Taiwan and China) also have public mental health systems. Thus, a study of hospitals run by the US Veteran's Administration is relevant in that care is both financed and delivered in the public sector. Further, while most veterans are male, the staffs providing their care are not. Finally, as concerns over cost containment grow, care systems domestically and internationally will face questions about the compatibility of treatment strategies such as psychosocial rehabilitation and increasing pressures for productivity. Such conflicts are especially likely in publicly sponsored delivery systems where providers are removed from direct market pressures.

\section{Appendix. Work Characteristics Scales}

(1) Relations with patients:

(a) The work that I do with patients is not very rewarding.

(b) In my job I often get to see patients improve.

(c) It is professionally stimulating to work with my patients.

(d) I enjoy working with the kinds of patients I work with.

(e) My patients appreciate my efforts.

(f) This job provides a challenging mix of patient diagnoses and problems.

(2) Relations with co-workers. 
(a) The people I work with take a personal interest in me.

(b) My co-workers appreciate my efforts.

(c) The people I work with are excellent mental health professionals.

(d) I have a strong feeling of trust in the people I work with.

(e) My co-workers have a strong interest in helping their patients.

(3) Workload:

(a) I have so many patients that I cannot give each one the care he/she needs.

(b) The amount of paperwork I have to do interferes with patient care.

(c) There is never enough time to get my work done.

(d) I have too much work to do everything well.

(4) Job hazards:

(a) My job is physically demanding.

(b) My job often exposes me to hazardous conditions (e.g. assault by a patient).

(c) The physical work involved in my job (e.g. lifting patients) exposes me to potential injury.

(5) Pay and rewards:

(a) Compared to others in my field who do not work for the VA, I am paid quite well.

(b) I am not rewarded fairly considering my education and training.

(c) The fringe benefits on this job are good.

(d) Given the amount of work I do, my pay is too low.

(e) I am rewarded fairly considering the responsibilities that I have.

(6) Role clarity and expectations

(a) I have enough authority to do my job.

(b) On my job, taks and responsibilities are clearly defined.

(c) This job gives me considerable opportunity for independence and freedom in how I treat my patients.

(d) Most of the time I know what to do on my job.

\section{References}

1. Stein LI. Innovating against the current. In: Stein LI, ed. Innovative Community Mental Health Programs: New Directions in Mental Health Services, Vol. 56. San Francisco, CA: Jossey-Bass, 1992, 5-22.

2. Witheridge TF, Dincin J. The Bridge: An assertive outreach program in an urban setting. New Directions Mental Health Services 1985; 26: $65-76$.

3. Mechanic D and Aiken LH. Capitation in mental health: Potentials and cautions. In: Mechanic D, Aiken LH, eds. Paying for Services. Promises and Pitfalls of Capitation. San Francisco: Josey-Bass, 1989.

4. Hu TW, Jerrell J. Cost effectiveness of alternative approaches of treating severely mentally ill patients in California. Schizophrenia Bull. 1991; 17: 461-468.

5. Lehman AF. Capitation payment and mental health care: A review of the opportunities and risks. Hospital Community Psychiatry 1987; 38: $31-38$.

6. Christianson JB, Gray DZ. What CMHCs can learn from two states' efforts to capitate Medicaid benefits. Hospital Community Psychiatry 1994; 45: 777-781.

7. Feldman S, ed. Managed Mental Health Services. Springfield, IL: Thomas, 1992

8. Frank R, Gaynor M. State government choice of organizational structure for local mental health systems: An exploratory analysis. In: Hu T, Rupp A, eds. Advances in Health Economics and Health Services Research 1994; 14: 181-196.

9. Craig TJ, Patterson DY. Productivity of Mental Health Professionals in a Prepaid Health Plan. Am. J Psychiatry 1981; 138: 498-501.
10. Hoge MA, Davison L, Griffith EEH, Howenstine RA. Defining managed care in the public sector. Hospital Community Psychiatry 1994; 45: 1085-1089.

11. Schinnar AP, Kamis-Gould E, Delucia N, Rothbard AB. Organizational Determinants of Efficiency and Effectiveness in Mental Health Partial Care Programs. Health Services Res. 1990; 25: 387-420.

12. Sorensen JE, Zelman W, Hanbery GW, Kucic R. Managing Mental Health Organizations with 25 Key Performance Indicators. Evaluation Program Planning 1987; 10: 239-247.

13. Foster B, Williams RB. Substitution of self-reporting for observing time spent on work activities by mental health professionals. Psychol. Rep. 1989; 64: 945-946.

14. Robichaud AM, Hamric AB. Time documentation of clinical nurse specialist activities. J Nursing Admin. 1986; 16: 31-36.

15. Coulton CJ, Keller SM, Boone CR. Predicting social workers' expenditure of time with hospital patients. Health Social Work 1985; 10: $35-44$.

16. Bachrach LL. Psychosocial rehabilitation and psychiatry: what are the boundaries? Can. J. Psychiatry. Rev. Can. Psychiatrie 1996; 41: 28-35.

17. Anthony WA. Switzer monograph: rehabilitation programs in the 1980s: laying the groundwork for the 1990s. Washington, DC: National Rehabilitation Association, 1988.

18. Anthony WA. Psychiatric Rehabilitation: Key Issues and Future Policy. Health Affairs 1992; Fall: 164-171.

19. Anthony WA, Cohen MR, Farkas MD. Psychiatric rehabilitation Boston: Center for Psychiatric Rehabilitation Boston University Sargent College of Allied Health Professions, 1990.

20. Alexander JA, Lichtenstein R, Oh H, Ullman E. A causal model of voluntary turnover among nursing personnel in long-term psychiatric settings. Res. Nursing Health 1998; 21: 415-427.

21. Gaynor $\mathbf{M}$ and Gertler P. Moral hazard and risk spreading in partnerships. RAND J. Econ. 1995; 26: 591-613.

22. Alexander JA, Lichtenstein R, D'Aunno T, McCormick R, Muramatsu N, Ullman E. Determinants of mental health providers' expectations of patients' improvement. Psychiatr. Services 1997; 48: 671-677.

23. Finlay W, Mutran E, Zeitler R, Randall C. Experience, Attitudes and Plans: Determinations of the Productivity of Medical Residents in a Primary Care Clinic. Work Occupations 1991; 18: 447-458.

24. Endicott J, Spitzer RL, Fleiss JL, Cohen J. The Global Assessment Scales: A procedure for measuring overall severity of psychiatric disturbance. Arch. Gen. Psychiatry 1976; 33: 766-771.

25. Jones SH, Thornicroft G, Coffey M, Dunn GA. Brief Mental Health Outcome Sacle: Reliability and Validity of the Global Assessment of Functioning (GAF). Br. J. Psychiatry 1995, 166: 654-659.

26. Bryk AS, Raudenbush SW. Hierarchical Linear Models: Applications and Data Analysis Methods. Newbury Park, CA: Sage, 1992.

27. Hoffman D. An overview of the logic and rationale of hierarchical linear models. J. Management 1997; 23: 723-744.

28. Bryk AS, Raudenbush SW, Congdon R. Hierarchical Linear Modeling with the HLM/2L and AHM/31, Programs: User's Reference Guide (Software Version 3.01/2.0). Chicago, IL: Scientific Software International, 1994.

29. Jinnett K and Alexander J. Case management and quality of life: Psychosocial care for US veterans with serious mental illness. San Francisco, CA: American Sociological Association, 1998.

30. Ivey SL, Scheffler R, Zazzali JL. Supply dynamics of the mental health workforce: Implications for health policy. Milbank Q. 1998; 76: $25-58$.

31. Wright TA. Well-being, satisfaction and job performance: Another look at the happy/productive worker thesis. Acad. Management Proc. 1997; 4: 364-368.

32. Morris A, Bloom JR. Organizational and individual predictors of job satisfaction, organizational commitment and managed care attitudes among a sample of mental healthproviders in Colorado. San Francisco, CA: American Psychological Association, 1998.

33. Schaefer JA, Moos RH. Effects of work stressors and work climate on long-term care staff's job morale and functioning. Res. Nursing Health 1996; 19: 63-73.

34. Timko C, Moos RH. Determinants of the treatment climate in psychiatric and substance abuse programs: implications for improving patient outcomes. J. Nervous Mental Disorders 1998; 186: 96-103.

35. Bodenheimer TS, Grumbach K. Understanding Health Policy: A Clinical Approach. Stamford, CT: Appleton and Lange, 1995. 